\title{
Alcohol causes epigenetic changes in hepatic stellate cells
}

"Alcohol directly stimulates epigenetic changes in hepatic stellate cells (HSCs) and augments their acquisition of fibrogenic characteristics," reports Jelena Mann, corresponding author of a study published in the Journal of Hepatology.

Chronic alcohol consumption can cause liver disease directly, as well as contributing to disease progression from other causes, such as viral hepatitis. HSCs are the major regulators of liver fibrosis. "We wanted to know if alcohol can augment the fibrogenic

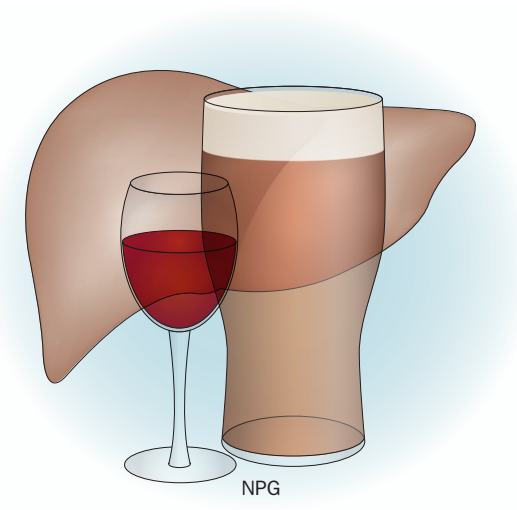

properties of HSCs," explains Mann. The researchers had previously shown that epigenetic signalling is important in HSC transdifferentiation and fibrogenesis. In addition, it is already well known that alcohol can cause epigenetic changes in liver tissue; however, the team wanted to establish whether alcohol directly affects epigenetic changes in HSCs in particular.

The expression of fibrogenic genes and epigenetic regulators was measured in HSCs undergoing transdifferentiation whilst incubated with alcohol. The researchers also used an in vivo model of alcoholic liver disease in mice, from which they isolated HSCs and performed similar molecular studies.

The most important finding was that alcohol has a direct effect on epigenetic changes in HSCs, enhancing their fibrogenic properties. For example, expression of extracellular matrix proteins, such as elastin, was increased. Mann and colleagues also found that alcohol stimulated the activity of MLL-1, a histone-modifying enzyme. When activated, this enzyme is recruited to the regulatory regions of fibrogenic genes where it makes the histone 3 lysine 4 trimethylation (H3K4me3) modification, which marks genes for active transcription. "We were already aware that this histone mark is generated during HSC transdifferentiation," explains Mann. "However, our new work discovered that this mark is further enhanced across the genome in cells exposed to alcohol.” These findings were confirmed in vivo.

"There are existing drugs that effectively inhibit MLL-1 that are being trialled in cancer," says Mann. "In future research we will determine the ability of these drugs to block alcohol-induced fibrosis in experimental animal models." The researchers hope that these preclinical studies could pave the way for targeting MLL-1 to prevent progressive liver disease caused by alcohol.

Isobel Leake

Original article Page, A. et al. Alcohol directly stimulates epigenetic modifications in hepatic stellate cells. J. Hepatol. doi:10.1016/j.jhep.2014.09.033 